\title{
Human System Interface Design and Operators Cognitive Strategies
}

\author{
P.V.R. Carvalho ${ }^{*},{ }^{1}$ I.J.A.L. Santos ${ }^{1}$ and M.C.R. Vidal $^{2}$ \\ ${ }^{I}$ Department of Instrumentation and Human Reliability, Nuclear Engineering Institute, Brazil \\ ${ }^{2}$ Luiz Alberto Coimbra Institute, Federal University of Rio de Janeiro, Brazil
}

\begin{abstract}
This paper focuses on the influence of Human-System Interface (HSI) design on nuclear power operators' actual work. During plant normal operation, operators' work comprises activities like monitoring, the use of procedures, cooperation mechanisms, and cognitive strategies for problem solving and decision-making. The work is characterized by constant changes in the focus of attention and the dynamics of conflicting activities. This paper explores the relationships between the operators' cognitive strategies and the constraints imposed by the HSI. The method employed for data collection was a field study during the operators' actual work in the plant control room. Our findings show that the HSI introduces background noise in the plant operation that shapes actions and decisions made by the operators.
\end{abstract}

\section{INTRODUCTION}

Nuclear power plant (NPP) control room operators observe and manipulate an extremely complex system. Their work situation requires walking along a large control panel, taking readings from gauges and adjusting knobs and levers. Vicente and colleagues already demonstrated that what makes monitoring difficult is the need to identify and pursue relevant findings against a noisy background [1]. In this research, we show that the Human-System Interface (HSI) is one of the most important noise sources during NPP operators' activities. Therefore, HSI design has significant implications for the safety of nuclear power plant, because they affect the mode that operators search for information and act to control the plant systems.

After the Three Mile Island (TMI) accident, where HSI and procedures design played a major role, NPP regulators around the world delivered recommendations to NPP designers and owners to use Human Factors Engineering (HFE) in the design and modernization of control rooms [24]. However, most of the modernization processes of instrumentation and control room systems have been driven to a large extent by the technology. The imagination of designers is stimulated by the available technology, and this often leads to solutions that look promising but in the long term may be less advantageous than originally thought.

An example of this technology-driven approach is shown in Fig. (1). When the old analog controllers of the turbine control could not be replaced due maintenance problems, a new computerized turbine control system was purchased. Although the new system performed its functions well, the installation of computer screen and keyboard along with the analog instruments in the hardwired panel brought some human-system interaction problems.

We also noticed that in HFE guides, human-performance issues are defined according to the tasks prescribed in

*Address correspondence to this author at the Department of Instrumentation and Human Reliability, Nuclear Engineering Institute, Brazil;

E-mail: paulov@ien.gov.br operational procedures. Contrary to the modern ergonomics approach, these guides take for granted that the prescribed tasks would match the actual operators' work activities [5]. Therefore, the complexity of monitoring activities is not fully taken into account, because there is no information about the actual cognitive strategies used by the operators to regulate their activities.

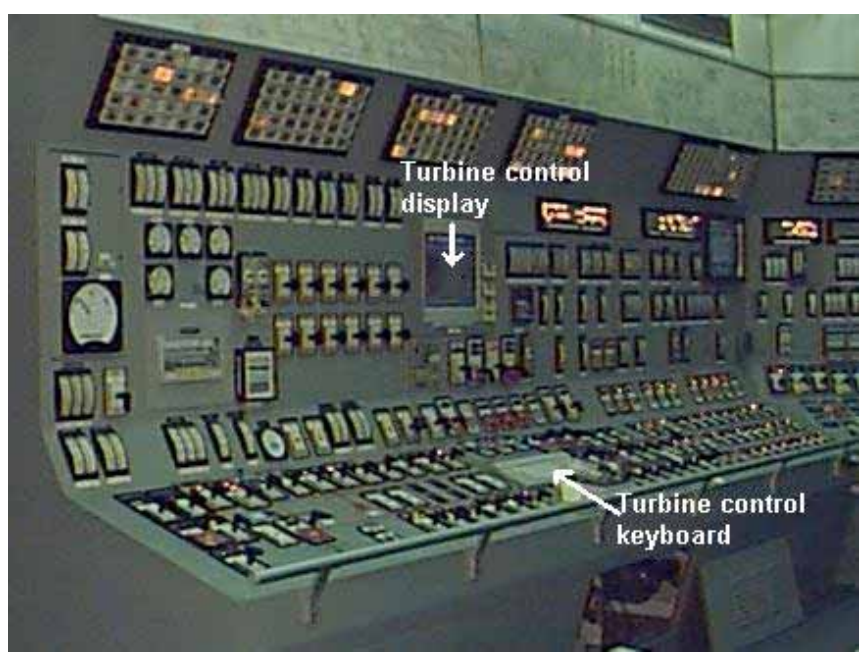

Fig. (1). Turbine display and keyboard together with analog instrumentation.

Faverge [6] established early that operators regulate their actions to achieve their objectives in the control of their plant. Hence performance variability is a normal behaviour in the operation of complex systems. In this conceptualization, actual work includes formal tasks, executed following procedures, together with some complementary activities developed by the operators during their daily work with the system. These activities emerge when operators have to face microincidents [7], poor interface designs, failures in equipment, incomplete procedures, time pressure, and other variations in the operational context - a complex and consequently non-predictable phenomenon. The regulations developed by the operators are based on cognitive strategies that are strongly shape by HSI design. 
The main objective of this research is to understand how HSI design influences the cognitive strategies developed by NPP operators during their normal work in their NPP control room.

\section{METHOD}

It is not easy to understand the interaction between humans and technology inside a complex organization, in their actual work environment. Humans' ability to adapt their behaviours to the specific environment, in which they have to perform their tasks, introduces a very difficult problem for research in human cognition. Indeed, human-cognition research would be easier if humans display the same behaviour in laboratory settings as they do in their actual workplaces. However, humans have an adaptive characteristic that changes their behaviour according to the task environment [8]. Humans also change their behaviour with the accumulation of experience and knowledge. In most complex system domains, tasks are often highly dynamic and complex [9], and human operators are experienced (in this study, the senior nuclear power operators had more than ten years of work experience). Therefore, the only way to shed some light on cognition in complex domains is to understand how operators' actual work is done, using field studies.

In this research, a field study was used to understand how nuclear power plant operators use the HSI in a real plant. The field study aimed to provide a means to understand how expert human operators achieve successful performance in a complex and event-driven task domain: managing the operation of a nuclear power plant. The field study carried out in the nuclear power plant's control room used direct observations to collect data. Direct observations led to a phenomenological account of the work activities in the NPP operation and a set of general patterns of behaviour and decisionmaking [10]. Video- and audio recording allowed a detailed study of the verbal protocols and implicit communication patterns [11] - focused on the anticipatory nature of behaviour, and substantiated the general findings of the direct observations.

The operators' activity analysis during an actual work shift was carried out during two different operation modes: start-up and shutdown. The data obtained were writings (field notes), filming, records, photographs, interviews with the operators, and read-out of operators' interactions. Five different operators groups were observed over three day shifts for a total of approximately twenty-four hours of observations. The goal was to obtain a detailed understanding of operators' monitoring activities, elucidating the operators' strategies utilized to solve problems. The confidence between ergonomists and operators built up during previous informal contacts, and the presence of the analysts inside the control room led to the use of more intrusive methods, including hot-reports, and thinking-aloud verbalizations during operators' work.

Beside the actual work observations, structured observations of the operators' activity in accident scenarios were carried out during the operators' training in the plant's fullscope simulator.

The observations procedure consisted of three phases: data collection, data preparation and data analysis.

\section{DATA COLLECTION}

Three video cameras inside the control room and micro recorders in the pocket of the four plant operators were used. Due the shared characteristic of the operators' activities and the dimension of the control room, we used four analysts inside the control room, two of them with a nuclear operation background, and another two with extensive experience in ergonomic work analysis in other industrial domains. We chose the planned shutdown and start-up of the power plant as the observation periods because during these periods the operators' workloads are higher (they have to perform control actions to change the plant state). Very brief interviews were carried out during work intervals. To probe some decisions more closely, we asked the operators about the cues they used to make their situation assessment, the goals in particular parts of the procedure, whether any other courses of action were considered when making a particular intervention, and whether the situation faced reminded them of any previous experience.

\section{DATA PREPARATION}

Data preparation was conducted on two data sets. This first one comprised the transcriptions of the operators' speech in chronological order, identifying all the people involved in the conversations. The second data consisted of the operational events in which the operators' used special cognitive strategies to overcome HSI-information problems. To identify these events, we used analysts' field notes, the first data set (transcriptions of operators' speech) and videotapes. In a first step, we identified all events perceived by each analyst (because each analyst was in closer contact with one specific operator, the perceptions of the events of each analyst could be quite different). In a second step, the four event tables obtained in the first step were placed together, and the ergonomists discussed their views on the development of events. At the end of this procedure we produced one final table with the chronological description of all events perceived by the analysts. After that, we searched for all verbal protocols related to each event since we started our observations (some events discussions related to the situation were noticed before the event, during the shift changeover, for example).

\section{DATA ANALYSIS}

The data formed by the final events table and the transcriptions of the operators' speech in chronological order were analyzed using the principles of protocol analysis [12] and content analysis [13]. The aim was to reduce the complex verbal protocols to accurate descriptions of the actions taken by the operators. Our aim was the identification of the cognitive strategies the operators used to cope with the problems in the HSI.

\section{THE NUCLEAR POWER PLANT}

This kind of applied ergonomic research emphasizes the importance of the work environment, which is described in this section. The site for the nuclear power plant is located on the South Atlantic coast in Brazil. It is a $1350 \mathrm{MW}$ nuclear power plant. The reactor is of the pressurized water type, PWR, with four reactor coolant loops. The primary plant is divided into the reactor system and the reactor coolant system. The reactor coolant system consists of four iden- 
tical coolant loops, including steam generators, reactor coolant pumps, reactor coolant pressurize system and reactor coolant pressure relief system. The reactor coolant pressuriser system consists essentially of the pressurise and the safety valves. The reactor coolant pressure relief system consists of the pressure relief tank.

The main control room area comprises the main control room, the equipment room, the process computer room and further rooms. In the main control room, the operators monitor and control the nuclear process, recognize disturbances that affect safety systems and maintain the plant in safe conditions. The main control room is subdivided into sections. The sections are: master control console, auxiliary control console, communications area, recording area, documentation area and personnel facilities area. The master control console consists of the main control desk (CWA) and the information desk (CWB). The master control console is shown in Fig. (2).

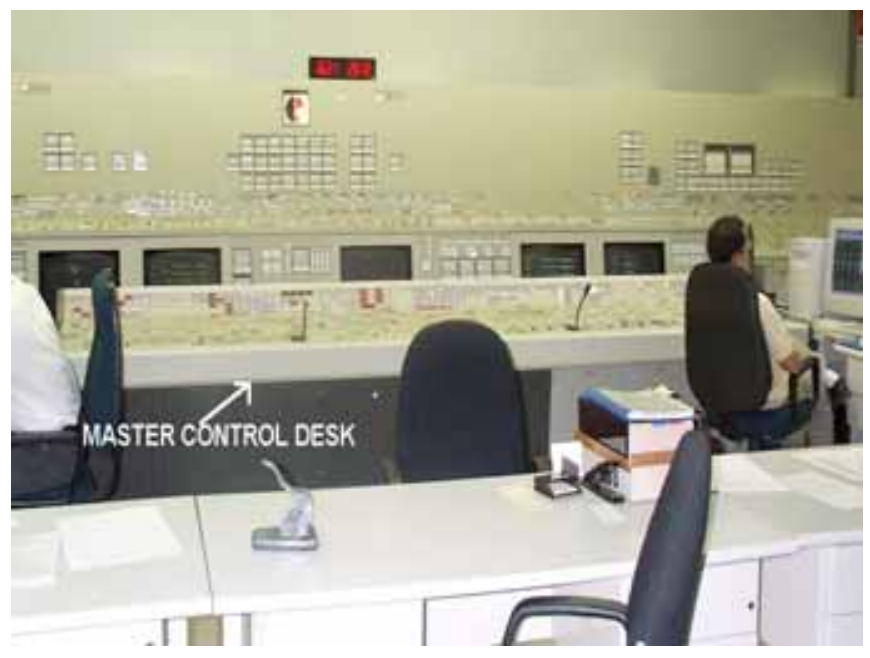

Fig. (2). Master control desk (CWA).

The operators are sitting on normal office roller chairs and are free to move all along the desk. Through the master control desk, the operators control the plant. The design of the master control console assures hand access to control tiles on the desk by the operators. The control panels use the mosaic technology, in which the traditional hard-wired meters, strip-chart recorders and control devices (start/stop pushbuttons) are mounted according to the functional diagrams of the various plant systems. The Supervisory Computer System (Fig. 3) makes available to the operators many monitoring functions, so that a better awareness of the plant status is achieved.

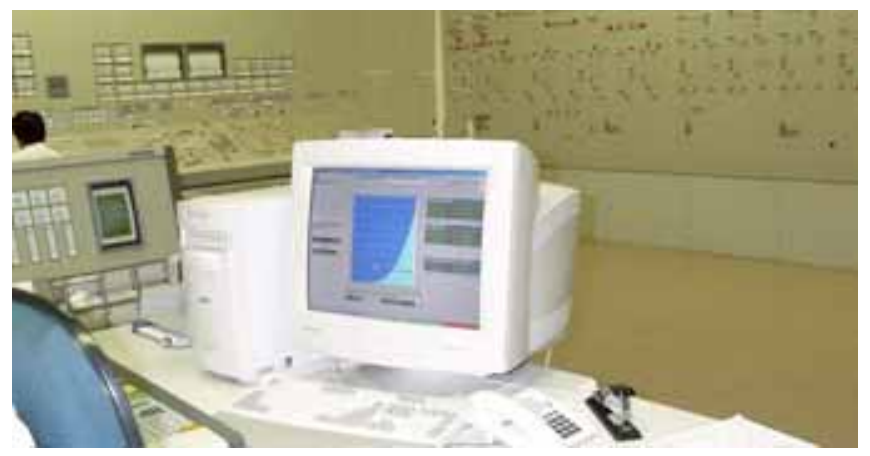

Fig. (3). The supervisory computer system.
Two independent alarm systems operate simultaneously: the hardware alarm system and the software alarm system. Illuminated desk tiles and acoustic signals deliver the hardware alarm system information. The information given by the software alarm system appears as an alarm list on the alarm video monitor in the control panel.

\section{THE CONTROL ROOM CREW}

The control room crew controls the power plant. Each shift crew of the main control room is normally composed of the following:

- one shift supervisor (SS), who is the responsible for the safe and efficient operation of plant during his shift;

- one shift foreman (SF), who reports to the shift supervisor, is the responsible for supervising the actions of the plant operators;

- $\quad$ one reactor operator (RO), who is the responsible for control the reactor and the safeguards system in a safe and reliable manner according to detail procedures. In off-normal situations, he will initiate actions until receives assistance from his supervisor;

- one secondary operator SCO, who is the responsible for the operation of the secondary plant control board and the operations requested by the load dispatch;

- one auxiliary or panel operator PO, who is the responsible for the monitoring the auxiliary equipments boards.

The operators' work is based on written procedures. The plant procedures are written on paper in English, which is not the operators' mother tongue. All the plant procedures have the same basic format. It begins with a flowchart, where the main event and operator actions are indicated. These actions are expanded in new flowcharts in a recursive way. Finally, the detailed instructions for each manual action and checklists are presented. The operators must fill out the blanks in the checklists with the detailed actions they carried out during the execution of the operational task.

Within this recursive-exploded structure, the operators have to browse the paper procedures continually, going from general diagnostic flow charts to the detailed manual actions and vice-versa. The foreman is in charge of reading (and browsing) the corresponding procedure to the $\mathrm{RO}$ and $\mathrm{SCO}$ during the operations. The RO and SCO confirm the instruction received, look at controls and act if needed. Finally, they inform to the foreman of the action taken.

\section{RESULTS}

Table 1 presents some events and the corresponding verbal protocols. In these events, the HSI is the main source generating noise.

\section{HSI AND OPERATORS' COGNITIVE STRATEGIES}

The first event, the nuisance alarms occurred mainly because the alarm system was not designed to consider the different plant operational states (start up, normal operation, and shutdown) to suppress some alarm signals according to the plant operational mode. Therefore, just after the 
Table 1. Events and Verbal Protocols

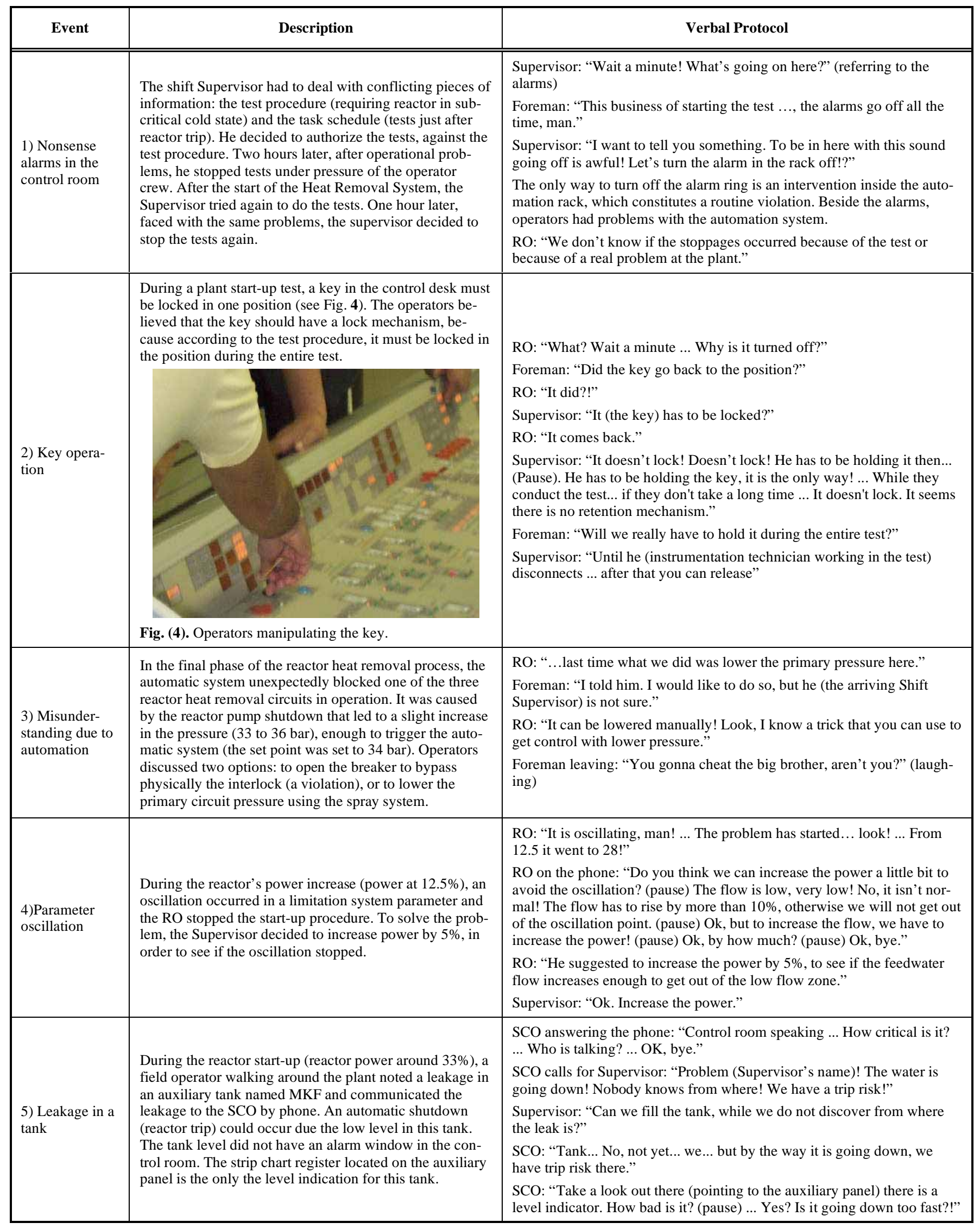


beginning of the instrumentation tests the control room was full of nuisance alarms. The only way to turn off the alarm ring is an intervention inside the automation rack, which constitutes a routine violation. However, this violation will clearly help the operation, since the reactor is already tripped and the nuisance alarms due the tests are disturbing the operation. However, this was not the only problem facing the operators at the time. The automation system uses the same signals used by alarm system and spurious blockades in important systems, such as the Reactor Heat Removal System, were in progress.

In Event 2, a simple question about the operation of a key brought much noise to the operation. The operators were not sure about the mode of operation of the key. They moved the key many times, expecting that the key had a locked position. However, the key did not have a retention mechanism, and the operator had to be holding the key for about $30 \mathrm{~min}$ utes during the entire test.

In the third event, the noise appeared after a pump shutdown that caused a slightly increase in the pressure of the primary circuit (33 to $36 \mathrm{bar}$ ). This pressure increase triggers the automatic system (the set point is in 34 bar) that turns off the reactor cooling circuits. This occurred at the end of the shift and operators from two operation crews discussed the problem and how to restart the cooling circuits. They discussed two options: opening the breaker to bypass the interlock physically, or lowering the primary circuit pressure using the spray system. This event exemplifies difficulties caused by the design of the automation system. The unexpected block in the heat removal process forced the operators into a complicated diagnosis process to restore the cooling capability of the system.

In event 4 , the noise came from an unexpected parameter oscillation during the plant start-up. The RO immediately stopped the power increase, reported the problem to supervisor and asked for the help of the instrumentation technician. The oscillating parameter is composed of many signals. One of them is the feedwater flow, which was very low. The instrumentation technician recognized a pattern, in which small variations in a low flow may result in spurious value signals, inferring that this might be the cause of the oscillation. The low flow could only be increased by an increase in the reactor power - but the increase in the reactor power was stopped because of the oscillation problem, creating two conflicting conditions. The decision to increase the power by $5 \%$ (another violation of routine, since no formal procedure considers the possibility of raising a 1300 MWE reactor by $5 \%$ just to see when oscillation stops) was successfully initiated by the operator crew.

In the last event, the identification of leakage in the tank by the control room operators was not easy. There was no alarm sound and the strip chart recorder that indicated the tank level was located in the auxiliary panel, far from the main control desk. The leakage was detected by visual inspection in the area, and might cause a plant automatic shutdown.

The design of the HSI and instrumentation, control and automation systems added background noise in the operation because of the following.
1. HSI design: difficult display visualization, inadequate position of strip chart registers, conflict between test procedures and the functionality of controls and indicators.

2. Instrumentation, control, and automation systems design: difficult for the operators to know what the automation system was doing.

3. Alarm system design: the alarm system did not support the different plant operational modes.

4. Communication system design: there were positions in the plant where people could not contact or be contacted by the control room. People needed to interrupt their work in the external areas and to answer the phone calls from the control room calls (the initial call from the control room was made using loudspeakers in the plant areas, and the worker called had to walk to the nearest phone cabinet to call the control room by phone).

The difficulties in display visualization are exemplified in Fig. (5), where a reactor operator struggles to see the entire display from his seated position and the panel operator is in an inadequate position to look at the strip chart recorder on the panel.

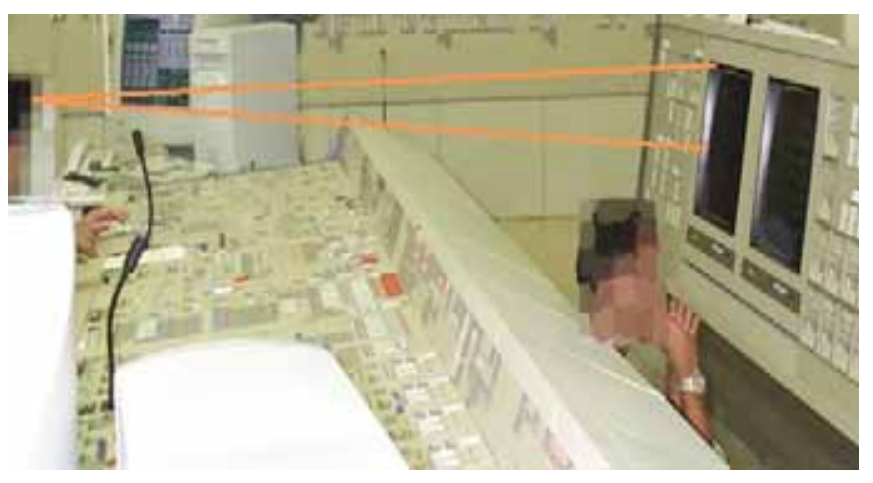

Fig. (5). From his position the operator cannot see the entire display.

The automation problems were related to the lack of adequate operational support to access automation system status. Plant automation was based on a complicated net of automatic sequence control modules used to control and safeguard systems, subsystems and equipment. Some of them were manually started/phased like reactor heat removal circuits, turbine synchronization, and others were automatically started after a reactor shutdown like emergency power sources and start of emergency core cooling. In situations when some system failed, operators struggled to identify in what part of the control sequence there were problems. To access automation system status, the operators only had the criteria display, in which they could see a logic diagram that indicated the status of each logical input/output of each automation building block. The operator's task was to integrate all this information, from equipment to system level, to construct a meaningful picture about what the automation was really doing.

The alarm system design did not consider the different operational modes of the plant (e.g. start-up, shutdown, and normal operation). Thus, during the change of operational modes, the operators had to cope with many spurious and 
nonsense alarms and they did not have the possibility to bypass specific alarms or group of alarms, without committing a procedure violation.

Voice communication links were frequently used to contact field operators, maintenance, instrumentation and engineering people. The control room used a conventional phone system, a broadcast system with loudspeakers spread around the plant, and the intercommunication system that connected the control to some specific areas of the plant. Radio and pagers were not allowed due to possible electronic interference with instrumentation and control systems. When the control room needed to talk with some field operator, a call was made using the general broadcast system and the field operator had to go to the near intercommunication system cabinet available to talk with the control room. We observed many difficulties in this communication routine because there were some blind points in the plant (no loudspeaker, or to noisy to be hear), and because the field operator had to stop his work to answer the control room call.

\section{OPERATORS ASSESSING THE PLANT SITUATION}

The aim of NPP's operators is to construct a mental model that matches the dynamics and evolution of the situation. This mental model is constructed by a continuous comparison of the current plant situation with the anticipation of the situation in the near future to plan required actions. After Endsley [14], this special type of mental representation is called Situation Awareness (SA). To achieve an adequate $\mathrm{SA}$, the operator must understand the current plant situation, monitor the various plant panels and other information sources (operational procedures, task plans, engineering documents, other people information), and anticipate the process evolution, to be able to control the process acting on the human-system interface. Therefore, the operators' work activity requires active monitoring and control strategies to cope with workload variations, maintaining SA that is paramount to ensure that the mental picture remains consistent with the actual situation.

When an operator becomes aware that the plant situation is different from the expected, his attention turns to information related to this difference. He has to understand the interface signals (as opposed to simply identifying the presence or absence or threshold overruns, which are a matter of indices or signals). Gathering and analysing the information related to the abnormal situation becomes the focus of the operators' attention. There is the moment when the operator concentrates on a single event, when he quickly excludes other information from his field of consciousness, and when he sets his mind to taking the meaning of the information he is given - something he can do only if he concentrates. It is reasonable to assume that there is a strong relationship between reading activity - that is, reading instruments and displays - as well as verbal and phoned communications, and a temporary mind-set in which the attention is focused on a single event or series of events, temporarily inhibiting and excluding everything else. It is precisely this sort of activity that dominates operation activity.

This reading/understanding activity and the search for meaning depends on the HSI design and constitutes a cognitive closed loop that enables the continuous generation of problem-solving strategies. It is likely that these strategies are mainly based on condition-action rules that are created on an ad hoc basis, supported by operators' tacit knowledge, mental model and underlying assumptions.

There are constant changes in focus according to the dynamics of the plant situation. At every moment, the operator has to discern precisely what he perceives in the plant panels, so he restricts his field of visual perception and attention focus to some specific part of the interface. In most cases, the reading of signs leads, both semantically and spatially, to additional information, but also makes move the agent physically to another point in the control room and to another kind of reading - as it occurs when the agent reads a value off in a display - or it can take him to a document (e.g. procedure, P\&I diagrams, logical drawings and alarm list), which must be pulled from its classification system and thumbed through until the right sheet is found. There is then a new focus of attention, but the operator must maintain his awareness all the time needed to cope with the situation. These constant changes in attention focus to keep situation awareness must be managed by the operators' working memory, which is a cognitive issue, especially important in analog HSI designs.

\section{ROUTINE VIOLATIONS AND HSI DESIGN}

The image that operators construct about the system (their mental models) and what will happen in the near future (situation awareness) played a significant role in the success or failure of the selected course of action. For instance, during an instrumentation test, the crew was unable to obtain an accurate picture of the relation between the tests and the automation system. Even if there was no safety problem (they were aware of this since they knew that the reactor had been turned off), alarms and blockades jeopardized the operation. They committed a violation [15] by turning off the alarm buzzer inside the instrumentation panel to make the operation easier under the circumstances - with the nuisance alarms and the reactor in shutdown mode. Such a strategy was shaped by the alarm system design that did not support alarm suppression according to the operation mode of the plant.

During Events 3 (reactor heat removal circuit blockade after pump shutdown) and 4 (a parameter oscillation, for more details see [7]), the operators bypassed the automation system, another procedure violation (in Reason's [15] sense) that proved to be successful. It is, therefore, of paramount importance for the safety and efficiency of operation that HSI design supports the correct way to do things; otherwise routine violations will occur.

\section{CONCLUSIONS}

The results of this study confirm that HSI design has a paramount influence on operators' work activities. In this study, operators were dealing with a HSI design that requires many ad hoc adaptations (including hardware, e.g. turnoff the alarm buzzer, and rules, e.g. change in procedures) to enable the system to function according to the situation demands. This study suggests that HSI introduces background noise that shapes operators' actions. These actions, including the ad hoc adaptations, should not be interpreted as violations - even if they are against plant procedures - because such ad hoc adaptations, in situations that were not antici- 
pated by the system design are needed during operation. They emerge as cognitive strategies created by experts to cope with unexpected situations and responding to an express need for additional or different working practices or tools.

This study also indicates that human factors/ergonomics requirements for HSI design, evaluation and validation should be applied in the design process in which the system is produced, and in the system itself.

The methodology used was included field studies and observations of the operators' performance in an actual control room. Performance evaluation-based methods have to be used because the appropriateness of a given system can be demonstrated only by the quality of the people's interactions with the system in real work situations. Normally, performance evaluation is something that is carried out toward the end of a given design process, and modifications in the HSI are very difficult to implement. This research indicates that performance evaluation should be done as early as possible in the design process using simulators.

Even considering that is very difficult to say when the performance of the joint cognitive system is at an acceptable level, our evaluation has shown that the existing HSI introduces background noise in the operation. Much of this noise is related to the consideration of basic human/factors ergonomic design principles. Examples include the following:

- Indicators and control with information that are difficult to read (inadequate position, and distribution).

- Confusing and unstructured presentation of automation system status, leaving the task of searching and detecting deviations of normal operations to the operator, instead of directly showing deviations caused by automation system.

- Static information presentation where a presentation of past dynamics (e.g. trends) and future developments of process parameters (predictive information) would be required for an effective task performance.

- A mix of different media to present operational information - analog indicators and controls, digital displays and paper procedures - requiring different cognitive resources.

As expected, this study has shown that the design solutions used (alarm systems, procedures, and control desks) actually have effects on operators' performance. We claim that design solutions should be produced considering the appropriate use of the system, emphasizing that work practices in complex industrial settings should not be based on the notion of human as the weakest link in the system that has to follow strict rules to avoid "human errors" and "procedure violations". We need systems that support actions of human operators, and their ability to adapt and adjust to novel situations. To do so, systems must be designed considering that the operators and the usage of the system is taken account of in all the phases of the design process, from the design of process technology to the design of user interfaces, in a user-centred and activity-based design process.

This research also illustrates the idea that whether a violation increases and enhances the control over a given task depends on the extent to which the operator's situation awareness accurately reflects and predicts the system's present and future states of the process. This defines two potential areas for improvement: the rationale for the initial violation and the content of the mental model itself.

\section{ACKNOWLEDGEMENT}

This research project has been partially supported by the Fundação de Amparo a Pesquisa do Rio de Janeiro FAPERJ.

\section{REFERENCES}

[1] Vicente K, Mumaw R, Roth E. Cognitive Functioning of Control Room Operators - Final Phase. AECB 96-175. Ottawa: Atomic Energy Canadian Bureau - AECB 1997.

[2] International Atomic Energy Agency - IAEA TECDOC 565. Control Rooms and Man-Machine Interface in Nuclear Power Plants. Vienna IAEA 1990.

[3] International Electrotechnical Commission IEC 1772. Nuclear Power Plants - Main Control Room - Applications of Visual Display Units. U.S. 1995.

[4] U.S. Nuclear Regulatory Commission NUREG 700 (Revision 2). Human System Interface Design Review Guideline. Washington DC 2002.

[5] Santos IJA, Carvalho PVR, Grecco CH, Victor M, Mol AC. A Methodology for Evaluation and Licensing of Nuclear Power Plant Control Rooms: Nuclear Atlantic Conference, INAC; Santos, SP, Brazil, 2005

[6] Faverge JM. Le Travail en Tant qu'activité de Récupération. Bulletin de Psychologie, 1980, Tomme XXXIII, n. 314.

[7] Carvalho PVR. Ergonomic field studies in a nuclear power plant control room. Prog Nucl Energy 2005; 48(1): 51-69.

[8] Simon H. The Sciences of the Artificial. Cambridge: MIT Press 1969.

[9] Woods DD, Roth EM, Pople HE Jr. Modeling operator performance in emergencies. Proceedings of Human Factors Society 34th Annual Meeting, p. 1132-5, 1990, U.S.

[10] Carvalho PVR, Vidal MC, Santos IL. Nuclear power plant shift supervisors decision-making during micro incidents. Int $\mathbf{J}$ Ind $\mathrm{Er}$ gon 2005; 35(7): 619-44.

[11] Carvalho PVR, Vidal MC, Carvalho EF. Nuclear power plant communications in normative and actual practice: A field study of control room operators' communications. Hum Factors Ergon Manufact 2007; 17: 43-78, 6: 38-45.

[12] Ericsson KA, Simon HA. Protocol analysis: Verbal reports as data. Cambridge: Cambridge University Press 1993.

[13] Krippendorff K. Content analysis. An introduction to its methodology. London, UK: SAGE Publications Ltd 1980.

[14] Endsley MR. Toward a theory of situation awareness in dynamic systems. Hum Factors 1995; 37: 65-84.

[15] Reason JT. Human Error. Cambridge, England, Cambridge University Press 1990. 